\title{
Descripción sistémico-funcional y gramática multiregistro
}

\author{
Systemic-functional description and multiregister grammar
}

\section{Rosa María Gutiérrez Barrientos}

Universidad Católica Silva Henríquez, Santiago, Chile. Correo electrónico: rmgutierrez@uct.cl

El objetivo de este estudio es describir, desde el marco teórico metodológico de la Gramática Sistémico-Funcional, la realización lexicogramatical de las opciones de la red sistémica del Tema en español. Para identificarlas, se hará una revisión bibliográfica de gramáticas del español y de estudios lingüísticos, en general, sobre la función de Tema. Esta etapa será complementada con un trabajo de precorpus sobre un reducido conjunto de documentos, lo que proporcionará un acercamiento a la lengua en uso y le conferirá sustento empírico a la revisión bibliográfica. La caracterización, por su parte, se desarrollada sobre la base de las diferentes escalas de abstracción que propone la teoría: rango, eje, detalle en la red sistémica, continuum de la lexicogramática y probabilidad de ocurrencia. Los principales resultados muestran que la red sistémica de tema en español presenta cinco opciones: tema lógico, lógico topical, topical, intertopical y interpersonal.

Palabras clave: tema, gramática sistémico funcional, gramática descriptiva, gramática multiregistro del español.

In this article, we describe, from the theoretical and methodological framework of Systemic Functional Grammar, the lexicogramatical realization of the options of the systemic network of the Theme in Spanish. To identify them, we carried out a bibliographical review of grammars of the Spanish and of linguistic studies, in general, on the function of Theme. This stage will be complemented by a work of pre-corpus on a small set of documents, which will provide an approximation to the language in use and will award empirical sustenance to the bibliographical review. The characterization developed on the base of the different scales of abstraction that proposes the theory: range, axis(axle), detail in the systemic network(net), continuum of the lexicogrammar and probability of occurrence. The principal results show that the systemic network of theme in spanish presents five options: logical, logical topical, topical, intertopical and interpersonal.

Key words: theme, systemic functional grammar, descriptive grammar, multiregister grammar of Spanish.

\section{INTRODUCCIÓN}

Como sabemos, la gramática de la lengua entendida como ciencia se ocupa de estudiar qué recursos verbales emplean los hablantes para comunicarse (Huddleston, 
1965; Halliday, 2002a), es decir, para crear significados (verbales) con el propósito de intercambiarlos. Dado que estos recursos verbales son parte del fenómeno del lenguaje verbal humano, una gramática debería asentarse en una teoría acerca de este último y, a partir de ella, dar cuenta del funcionamiento de la lengua, que es el sistema que aglutina dichos recursos. A partir de esta idea, podemos reconocer fundamentalmente dos tipos de gramáticas. En primer lugar, están las gramáticas que, basadas en alguna concepción del lenguaje (estructuralista, funcionalista, pragmática), adoptan un enfoque eminentemente deductivo al describir. De este modo, los gramáticos suelen analizar y describir los fenómenos de la lengua basándose en teorías acerca del lenguaje, pero ejemplificando desde su propio conocimiento como hablantes o utilizando conjuntos de textos de pequeña extensión que no dan cuenta de la variedad implicada en el uso real de los recursos. Las gramáticas de este primer tipo pueden subdividirse, según el alcance de sus objetivos, en gramáticas normativas (e.g. RAE (1973)) o descriptivas (e.g. la dirigida por Bosque y Demonte (1999)) y, según el énfasis teórico subyacente, en funcionales (e.g. Hernández (1986)), generativas (Di Tullio 1997) o cognitivas (e.g. Gili Gaya 1979).

En segundo lugar, y en un sentido contrario al recién expuesto, frente a la necesidad de describir, precisamente el uso real, se han desarrollado estudios que toman un punto de partida inductivo y que utilizan las herramientas computacionales para trabajar la descripción de patrones de usos y comportamientos linguíísticos sobre la base de un amplio conjunto de textos tomados de la ocurrencia real (e.g. Biber, Conrad y Reppen 1998). Sin embargo, es frecuente que este tipo de trabajo no cuente con una sólida y unificada teoría sobre el lenguaje y que, en consecuencia, la situación se descompense en el sentido inverso al de las gramáticas del primer grupo. En este contexto, la Gramática Sistémico-Funcional (GSF de ahora en adelante) se posiciona como una gramática que contempla las dos potencialidades que hemos definido. Así, la GSF es una gramática que tiene un fuerte anclaje en el marco de una teoría acerca del lenguaje, la Lingüística Sistémico-Funcional (en adelante, LSF) y que, desde ella, ha construido sus propios métodos para la descripción inductiva de las lenguas. Estos procedimientos metodológicos se vinculan directamente con los principios teóricos subyacentes, como tendremos oportunidad de observar en la exposición de este trabajo. Al tomar esta perspectiva, se espera contribuir al desarrollo de una gramática del español que, sustentada en un sólido andamiaje teórico, se centre en el uso y, en este sentido, contribuya a la descripción multiregistro del español. Además, puede resultar ser un buen aporte al desarrollo de diferentes ámbitos. En primer lugar, y en términos generales, una descripción gramatical, cualquiera que ésta sea, amplía el conocimiento metalingüístico de los hablantes, permite conocer el estado de ella y sobre éste, desarrollar estudios contrastivo de la misma lengua (diacrónicamente) o entre lenguas (sincrónica o diacrónicamente). En segundo lugar, al estar la descripción sistémica basada en las instancias de uso, proporciona un tipo de gramática que marca el paso desde una centralizada o estandarizada a una contextual y descentralizada que considera las variaciones geográficas, sociales y de estilo (Martínez 1999; Caffarell et al. 2004). Desde aquí, resulta evidente que una GSF tiene importantes implicaciones en la didáctica de la enseñanza del español como lengua materna, así como lengua extranjera, por cuanto proporciona el espacio para el aprendizaje 
no prescriptivo de la lexicogramática, esto es, un aprendizaje desde la observación del uso de las configuraciones lexicogramaticales en textos reales, producidos por hablantes reales, frente a necesidades contextuales que también lo son, abriendo con ello la consecuente comprensión del lenguaje como un fenómeno del cual los hablantes y su cultura forman parte. Una GSF también podría servir de base para estudios psicolingüísticos del desarrollo del lenguaje, de la comprensión y de la producción del discurso, por cuanto, de una parte, los complementa desde una comprensión social y, de otra, sienta las bases para los recientes trabajos acerca de la corporeidad involucrada en las construcciones de significados que las personas configuramos y de la relación de éstas con el léxico y la gramática (De Vega 2005). Además, al trabajar sobre componentes semánticos, la GSF se constituye en el modelo gramatical que puede satisfacer de modo más acabado las exigencias de los analistas del discurso, tal como lo reconociera Fairclough (1995); en tanto que al ser los componentes semánticos postulados como universales, la descripción de las lenguas desde la LSF se presenta como especialmente apta para la traducción.

Una descripción sistémico-funcional opera con categorías teóricamente predefinidas, postuladas como universales (comunes a todas las lenguas), y con categorías definidas a partir de la descripción sistémico-funcional de una lengua determinada y, en consecuencia, particulares de esa lengua (Caffarell, Martin, Matthiessen 2004). Tal particularidad impone la necesidad de desarrollar descripciones específicas para cada idioma. Actualmente existen gramáticas sistémico-funcionales (GSF, en adelante) del inglés (e.g. Halliday, 1985) y del francés (e.g. Caffarell 1995; 2004), además de un amplio conjunto de trabajos que, con un afán menos ambicioso que el de elaborar la GSF de la lengua que estudian, abordan la descripción de la realización de alguno de los sistemas semánticos (i.e. ideacional, interpersonal o textual) de lenguas tales como el chino (e.g. Yang, MacDonald y Musheng 1995; Halliday y McDonald 2004), el alemán (e.g. Steiner y Teich 2004), el japonés (e.g. Teruya 2004), el vietnamita (e.g. Duc 2004), el español (e.g. Martínez 1999; Arús 2006; Gutiérrez 2007, 2008, 2010; Moss y Chamorro 2008; Moss, Natale y Oteiza 2010; Moyano 2007), etc. Estos trabajos, si bien han constituido un buen aporte para la aplicación de la GSF al español, se caracterizan, en su mayoría, por ceñirse de modo estricto a las categorías descritas por los lingüistas sistémicos para el inglés (en especial por Halliday (1985), Halliday y Matthiessen (2004) y Martin (1992)), tomando, en consecuencia, un carácter contrastivo que obvia los rasgos que particularizan a cada lengua. Este hecho pone en riesgo el carácter sistémico-funcional de la descripción y adquiere especial relevancia frente a la diferenciación entre lenguas disociativas como el inglés y el chino y lenguas flexivas como el francés, el alemán y el español, cuya descripción requiere un detalle (i.e. delicacy) que alcance el nivel morfemático.

En este estudio se aborda la descripción sistémico-funcional de las opciones que, en español, realizan léxica y gramaticalmente la función de tema. Se trata de un estudio en el que se sigue la metodología propuesta por Caffarell, Martin y Matthiessen (2004) para la descripción sistémico-funcional de las lenguas. Dos objetivos específicos se asocian a la descripción: identificar las configuraciones y caracterizarlas de acuerdo a las 'dimensiones sistémicas', i.e. metafunción, instanciación, estratificación, rango, continuum de la lexicogramática y eje y detalle en el marco de la red sistémica. 


\section{MARCO TEÓRICO}

La LSF es una teoría que retoma y pone en interrelación los postulados básicos y generales del estructuralismo. Lo que marca una especial diferencia entre LSF y las otras escuelas estructuralistas es el enfoque decididamente contextualista, enmarcado en los hallazgos seminales de Malinowski en la década del 20 (1984) y que posteriormente fueran desarrollados por Firth (1968a, 1968b, 1968c). En consonancia con ello, LSF propone tres modos de abordar, teórica y metodológicamente, la dependencia contextual del significado. Estos son: significado como elección, significado como (meta)función y significado como variación. En torno a cada uno de ellos se organiza la exposición en este apartado.

\subsection{SIGNIFICADO COMO ELECCIÓN: LA LENGUA COMO SISTEMA SEMIÓTICO}

Como sabemos, se comprende que la lengua y el lenguaje verbal son sistemas semióticos, es decir, sistemas cuya función principal es construir significados con el propósito de interactuar, con otros y con el mundo, satisfaciendo necesidades comunicativas (Halliday 2003a). En esta idea, la lengua se presenta como un conjunto potencial de recursos para significar ('proceso' en palabras de Hjelmslev, 1974). Los hablantes elegimos recursos desde el potencial y cada una de las elecciones va contribuyendo a la construcción de significado. Estas elecciones no son completamente voluntad del hablante, sino que están motivadas y condicionadas por el contexto a través del proceso de 'realización'. Por medio de éste, los estratos formantes de un sistema semiótico se vinculan entre sí de modo que las elecciones efectuadas en un estrato pasan a funcionar como el contexto o ambiente (realizacional) que motiva y condiciona las elecciones que pueden hacerse en el estrato inmediatamente inferior (Berry 1977; Halliday y Matthiessen 1999; Halliday 2003b, c; Matthiessen 2006, 2008).

Siguiendo la idea que Hjelmslev formulara en el año 40 (Hjelmslev 1974), la LSF reconoce una organización biestratal del sistema semiótico del lenguaje verbal (Halliday y Matthiessen 1999; Halliday 2003a; Caffarell et al. 2004). Dicha organización comprende un estrato de contenido y uno de expresión del contenido, el contexto y la lengua, respectivamente, tal como lo grafica la Figura 1, a continuación.

Figura 1

Estratificación del sistema semiótico del lenguaje verbal

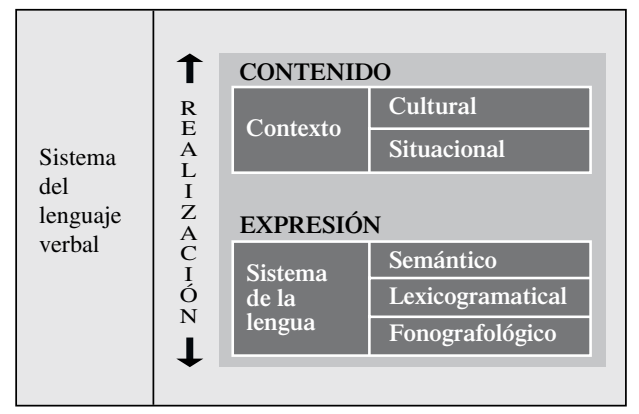


Así, decimos que el estrato del contenido o contexto motiva y acota las elecciones que pueden hacerse en el estrato de la expresión o lengua, i.e., el contexto le da 'forma a la lengua'.

Como se muestra en la Figura 1, cada uno de estos planos es en sí mismo otro sistema semiótico. En tanto que sistema semiótico, la lengua se diferencia de otros por presentar una organización tri y no biestratal. Lo que ocurre es que el plano del contenido se subdivide en otros dos: el estrato semántico y el estrato lexicogramatical (o de la gramática). Este último es el nivel que agrupa las opciones léxicas y gramaticales que los hablantes tenemos a disposición para construir significados (Berry 1977) y su descripción constituye el foco de este estudio. Para operar la descripción se hace uso de tres constructos teórico-metodológicos: la escala de rango, el continuum de la lexicogramática y la red sistémica. A través del rango, las unidades del estrato lexicogramatical (i.e. cláusula, frase o grupo, palabra, morfema) se organizan en una escala jerárquica y taxonómica (Halliday, 2002b, 2002c; Halliday y Matthiessen 2004) de acuerdo al lugar que ocupan en la estructura sintagmática (Halliday 2002b, 2002a; Halliday y Matthiessen, 2004), evidenciando cómo funciona una lengua, para construir el significado, en términos de constituencia, es decir, en términos de articulación de unidades menores en mayores. El continuum de la lexicogramática, por su parte, permite clasificar los patrones lexicogramaticales como más léxicos o más gramaticales, en el entendido de que la gramaticalización y la lexicalización son fenómenos que tienen lugar en el marco de la evolución onto, filo y logogenética de la lengua (Halliday y Matthiessen 1999, 2004) y, por tanto, no dependen exclusivamente de la realización de las categorías. Finalmente, la red sistémica, propuesta por Firth (1968a, 1968b, 1968c), es el constructo que permite formalizar el concepto de significado como elección, al presentarse como un conjunto de opciones o términos del sistema, con una condición de entrada, representando el ambiente o contexto en el cual las elecciones son efectuadas, y una de salida (Halliday 2003c), mostrando las posibilidades y probabilidades de 'instanciación'. La instanciación es un proceso que puede ser visualizado como una actualización paulatina del potencial de significado hasta hacerlo completamente observable en una 'instancia' o texto (Matthiessen 2008). Se trata de un proceso que se desarrolla en forma de una gradiente (i.e. cline) en la cual se reconocen a lo menos tres grados de abstracción de la instancia: texto, tipo de texto o tipo de instancia y registro, tal como se muestra en la Figura 2.

A través de la instanciación, se vincula el sistema con su manifestación observable, en nuestro caso, el texto. La representación sistémica involucra las dimensiones organizadoras de eje y detalle (delicacy). Desde el eje, las opciones son vinculadas tanto paradigmática como sintagmáticamente (Halliday 2002a, 2003d, 2003a). La dimensión paradigmática (i.e. eje vertical) presenta a las opciones como elecciones simultáneas o como elecciones excluyentes (Halliday 1985b, 2002a). La dimensión sintagmática (i.e. eje horizontal), por su parte, presenta las opciones sucesivas a medida que se avanza, a través de la escala de detalle hacia la instanciación (Halliday 2003d). Existe una correlación inversamente proporcional entre la escala de detalle y la idealización de una red sistémica, tal que, a menor grado de detalle, mayor grado de idealización de la red (Halliday 2002b). Tal correlación proviene del principio teórico que señala que el detalle (i.e. la delicacy) se va obteniendo a medida que se avanza hacia la instancia particular y concreta que es la que, finalmente, permitirá, 
Figura 2

Continuum de la instanciación

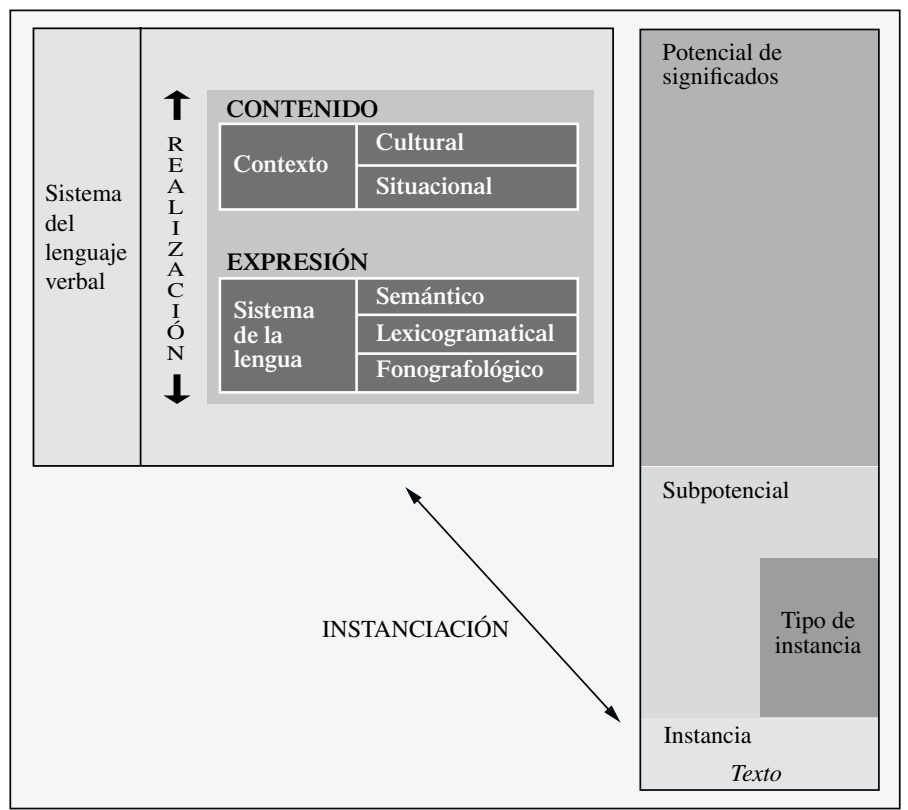

desde el punto de vista descriptivo, determinar la probabilidad con que cada una de las opciones de una red sistémica es actualizada o instanciada en el uso.

\subsection{SIGNIFICADO COMO VARIACIÓN: LA LENGUA COMO UN POLISISTEMA}

Para la GSF, el sistema se configura sobre la base de la observación de las instancias o manifestaciones concretas del potencial. Al operar la descripción del sistema desde los usos concretos, tal como lo postularan los estructuralistas de Praga, la idea de que una lengua constituya un sistema único es insostenible en el marco de la LSF. Lo que se acepta es que hay patrones lexicogramaticales más o menos regulares que tienen diferente probabilidad de ser instanciados en el marco de los distintos contextos de uso (Halliday y Matthiessen 1999). Esto no quiere decir que la GSF se preocupa de los contextos reales, sino que más bien de algunos rasgos que regularmente se presentan en ellos y que se asocian, regularmente también, a la variación en las elecciones que se hacen desde el sistema. El registro es el constructo teórico que permite dar cuenta de dicha relación a través de las variables que lo conforman: campo (i.e. acción social en la que la lengua está siendo usada), tenor (i.e. relación entre los participantes de un interacto de habla) y modo (i.e. modo de presentación del texto en el intercambio comunicativo) (Haliday 1982; Matthiessen 1993).

En efecto, el sistema es sistema de las probables elecciones que los hablantes harán en el uso de la lengua en contexto y, por tanto, una red sistémica solo se ha completado cuando se determina la probabilidad de ocurrencia o instanciación de cada configuración (Halliday 1991, 1992, 2005b, 2005d; Matthiessen 2006). Para 
esto se requiere observar un corpus amplio de textos tomados de la ocurrencia real y justificado en términos lingüísticos a partir de las variables de registro que son las que le confieren al conjunto su rasgo de subpotencial de significado, en el marco del continuum de la instanciación (ver Figura 2). Así visto, el corpus es más que una suma (o colección) de instancias; es una manera de observarlas desde el sistema y de comprenderlas, entonces, como un subpotencial (Matthiessen 1993; Halliday 2005b, 2005d; Halliday y Matthiessen 2004; Matthiessen 2008). Esto, a su vez, posibilita la comprensión del sistema como entidad teórica, más precisamente, la teoría de la instancia (Halliday y Matthiessen 2004; Halliday 2005a).

En el marco de LSF se reconocen y se emplean dos tipos de aproximaciones al trabajo con corpus. La primera de ellas, 'basada en el texto', potencia un trabajo sobre pequeños grupos de textos, con el fin de obtener un acercamiento detallado a las configuraciones que son objeto de la descripción. La segunda, 'basada en el corpus', potencia un trabajo sobre grandes cantidades de textos, con el fin de obtener una mirada más amplia, desde la probabilidad con que las configuraciones son usadas. Desde ambas perspectivas, el corpus es considerado como un medio para acceder a la teorización del sistema, es decir, como una metodología de trabajo (Matthiessen 2006).

\subsection{SIGNIFICADO COMO FUNCIÓN: LA HIPÓTESIS METAFUNCIONAL}

Finalmente, la tercera forma en que se manifiesta la dependencia contextual del significado es a través de la hipótesis metafuncional. Desde aquí, se comprende que el sistema de la lengua satisface funciones que provienen del contexto en que el sistema es usado. Dado que el contexto es uno de los estratos del sistema semiótico del lenguaje verbal, estas funciones no son externas al sistema, sino que, a través del proceso de realización, parte de él, y sobre su base se organizan todos sus niveles. Son básicamente tres: (i) ideacional o función de representar el mundo y la propia experiencia; (ii) interpersonal o función de interactuar con otros y (ii) textual o función de proveer de recursos para textualizar la representación del mundo. Desde la hipótesis, se postula que estas funciones son universales semánticos, asociados a la lógica de un interacto comunicativo, de modo que es posible reconocer que la metafunción ideacional se vincula a la tercera persona o aquello de lo que se habla; la metafunción interpersonal se focaliza en la segunda persona participante del interacto y la metafunción textual, en la primera persona o en quien inicia el interacto comunicativo. Se les llama metafunciones para significar que los tres sentidos en que el sistema verbal semiotiza la realidad forman parte del sistema y operan simultáneamente.

Las metafunciones se realizan a nivel lexicogramatical en la estructura funcional de la cláusula. Se trata de una estructura trifásica en concordancia con las tres líneas de significado que realiza: (i) la transitividad asociada a la metafunción ideacional; (ii) la interpersonal asociada a la interpersonal y, (iii) la textual, correspondiente a la metafunción del mismo nombre. Cada una de ellas está conformada por funciones diferentes: la transitiva está conformada por las funciones básicas de proceso, participantes de los procesos y circunstancias en que ellos ocurren; la estructura interpersonal lo está por las funciones de núcleo modal y residuo; y la estructura textual, por las funciones de tema y rema (Halliday 1982a, 1985; Matthiessen 1995; Halliday y Matthiessen 2004), tal como se muestra en la Figura 3, a continuación. 
Figura 3

Composición de las estructuras funcionales

\begin{tabular}{|l|l|l|l|l|l|}
\hline Estructura funcional & \multicolumn{2}{|c|}{ Funciones } \\
\hline Representación & participante & Proceso & participante & \multicolumn{2}{c|}{ circunstancia } \\
\hline \multirow{2}{*}{ Intercambio } & \multicolumn{2}{|c|}{ Núcleo modal } & \multicolumn{3}{c|}{ Residuo } \\
\cline { 2 - 6 } & Sujeto & Finito & Predicador & Complemento & Adjunto \\
\hline Mensaje & \multicolumn{2}{|c|}{ Tema } & \multicolumn{3}{c|}{ Rema } \\
\hline
\end{tabular}

Las estructuras funcionales, al igual que los componentes (metafuncionales) que realizan, son postuladas como universales y, en consecuencia, compartidas por todas las lenguas. Desde esta idea, en el estrato lexicogramatical, que constituye nuestro foco de atención, es posible reconocer que una cláusula presenta no solo una estructura sintáctica (del tipo Verbo + Grupo Nominal, por ejemplo), como la oración, sino que también y, de modo paralelo, presenta esta estructura funcional (del tipo proceso + participante, por ejemplo) de carácter trifásico.

El modo en que metodológicamente se da cuenta del significado como (meta) función es a partir del punto semántico desde el cual parte toda descripción de una lengua. Así, por ejemplo, en este estudio se toma por punto de partida a la metafunción textual.

\subsection{METAFUNCIÓN TEXTUAL: LA FUNCIÓN DE TEMA}

Como ocurre con casi todo el metalenguaje en lingüística, no es fácil acercarse a una definición de 'tema'. En primer lugar, 'tema' es un término que nos sirve para referir al tópico que se desarrolla en un texto (i.e. idea general tratada en un texto); asimismo, sirve para referir al tema de la acción social en el marco de la cual tiene lugar el intercambio comunicativo (i.e., tema como variable en el marco del registro); en la terminología de la Escuela de Praga (Tesis del 29), tema es 'la información que el hablante presume conocida por su oyente y en cuyo marco se intercambia un nuevo contenido, 'lo dado'; finalmente, tema también es un vocablo usado para nombrar la función que, en el marco de la estructura funcional de la cláusula como mensaje, realiza significados asociados a la formación del texto (Halliday 2004), en otras palabras, significados asociados a la metafunción textual. Específicamente, desde esta última conceptualización que es la que nos interesa, se sostiene que el tema da forma verbal a los propósitos comunicativos del hablante, constituyendo de este modo el marco que orienta al lector respecto de cuál es el instrumento verbal empleado en dicho intercambio. Así, por ejemplo, cuando escuchamos o leemos un enunciado como 'Lea' podemos fácilmente reconocer que el propósito comunicativo del hablante es explicar algún procedimiento y el instrumento verbal empleado es un manual o instructivo.

Desde la GSF, se han elaborado variadas hipótesis sobre la realización lexicogramatical de la función de tema (Enkvist 1973; Halliday 1985; Matthiessen 1995; Stainton 1993; Berry 1977; Halliday y Matthiessen 2004). Todas ellas coinciden en que, a nivel lexicogramatical, la función de tema se realiza en el elemento que ocupa 
la primera posición en una cláusula. En otras palabras, el orden de los elementos en la estructura sintáctica sería el recurso verbal empleado para realizar significados asociados a los focos y metas comunicativas que orientan el intercambio comunicativo. En este sentido, el tema es el punto de partida para el desarrollo del texto y corresponde a lo que el hablante pone en foco desde la perspectiva de la organización del texto mismo (Halliday 2005). Esto es lo que conocemos como sujeto psicológico (Halliday y Matthiessen 2004). Como bien lo apunta Gili Gaya (1979), este tipo de sujeto no depende solo de nuestro interés del momento, sino que de "factores o moldes ideales, esquemas expresivos, fórmulas de organización que hemos aprendido desde niños y que aplicamos por analogía a las frases, oraciones y períodos." (Gili Gaya 1979:7). En efecto, todos nuestros enunciados tienen formas típicas para la estructuración (verbal) de la realidad y estas organizan nuestro discurso de la misma manera que lo hace cualquier otra forma gramatical.

$\mathrm{El}$ contexto desde el que provienen las restricciones u orientaciones para la realización de la función de tema son estas formas cristalizadas de interacción verbal que constituyen verdaderos formularios o siluetas textuales que orientan la organización de la representación verbal del mundo (i.e. la resemiotiza), dándole forma de texto verbal a nuestros propósitos comunicativos. La variable de registro que permite describir la variación en la tematización es el modo. Esta última se refiere, de una parte, al modo de organización simbólica del texto, i.e. oral-escrito, de otra, a la organización discursiva presente en el mismo, i.e. argumentativo, narrativo, descriptivo, así como también al tipo de texto o género en el cual el modo de presentación y el modo de organización discursiva se manifiestan, tal como se observa en la red sistémica de la Figura 4 a continuación.

Figura 4

Red sistémica de la variable de modo

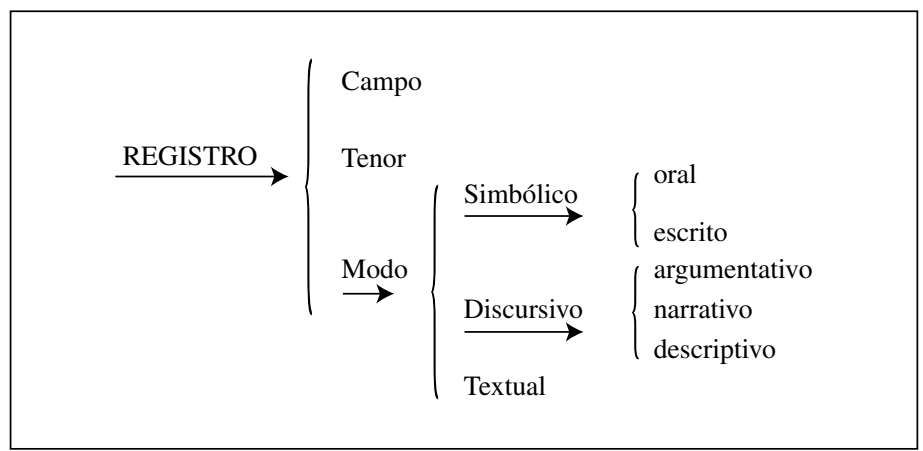

Como puede observarse en la red sistémica de modo, en la Figura 4, un hablante efectúa simultáneamente elecciones de presentación, modo de organización discursiva y tipo de texto. Son estas elecciones las que orientan y condicionan la selección del tema de una cláusula. 


\section{Metodología}

\subsection{OBJETIVOS}

El objetivo general de esta investigación es describir, desde el marco teóricometodológico de la GSF, la realización lexicogramatical de la función de tema a nivel de la estructura sintáctica en español.

La descripción sistémico funcional, según la metodología propuesta por Caffarell et al. (2004), involucra dos actividades: identificar y caracterizar. Cada una de ellas se constituye en objetivo específico de esta investigación. La redacción detallada de ellos se presenta a continuación:

a) Identificar, a través de una revisión bibliográfica y de una exploración de precorpus guiada por el corpus, las configuraciones lexicogramaticales que, en español, realizan las opciones de la red sistémico-semánticas del tema en español.

b) Caracterizar las configuraciones lexicogramaticales que, en español, realizan las opciones de la red sistémico-semántica del tema, a partir de su distribución en las escalas (de rango, eje y detalle), dimensiones y condiciones de una GSF.

\subsection{Procedimientos metodológicos}

En concordancia con los dos tipos de objetivos específicos que se pretenden, la descripción a desarrollar en esta investigación contempla dos etapas fundamentales: identificación y caracterización, tal como se presenta en la Figura 5.

Figura 5

Acciones implicadas en una descripción sistémico-funcional

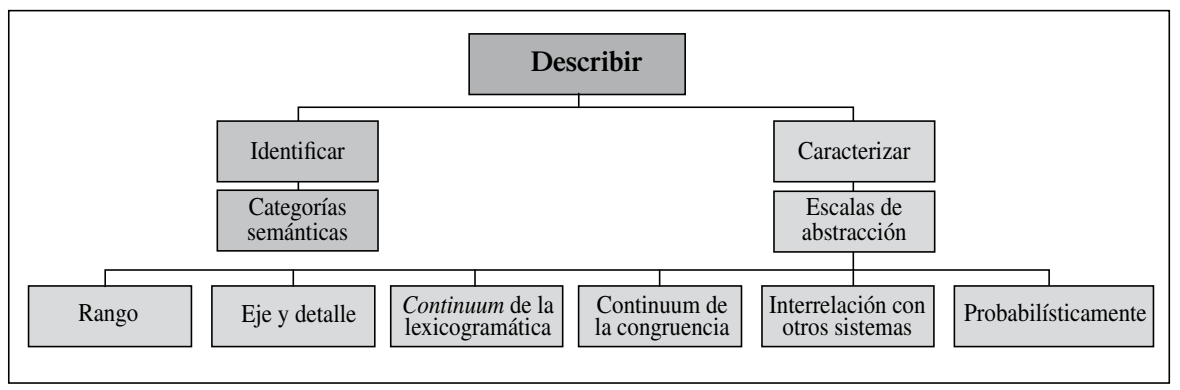

A continuación, se explicitan los pasos metodológicos y las variables asociadas a cada una de las etapas.

\subsubsection{Etapa de identificación}

Esta etapa permite satisfacer el primer objetivo específico de este trabajo (identificar las configuraciones) y contempla dos fuentes de datos. En primer lugar, un variado conjunto de gramáticas del español sin restricción de enfoque (normativas, funcionales 
y descriptivas) ni de tiempo. Sobre ellas, se realiza una revisión bibliográfica con la que se consigue retomar, en el marco de la descripción sistémico-funcional, la tradición de los estudios gramaticales del español. Para esta etapa se utiliza un muestreo teórico cuyo arranque está dado por cuatro contextos de observación, según el cruce de dos criterios, a saber: tipo de estudio y año de publicación. El primer criterio diferencia entre estudios gramaticales y estudios lingüísticos, a su vez, las gramáticas se subdividen en normativas, descriptivas y funcionales. El segundo criterio es transversal y considera las gramáticas y estudios cuya fecha de publicación es más reciente.

La variable que orienta la identificación de las configuraciones que, a nivel de la estructura sintáctica, realizan los significados asociados a la tematización de una cláusula, es la definición semántica del concepto de tema. Esta definición permite dar cuenta del 'significado como (meta)función'. Como se ha profundizado en el marco teórico, se entiende que el tema es el elemento que aparece en primera posición en una estructura clausular y se extiende hasta el verbo, incluyéndolo, pues en español no es posible disociar el elemento argumental de la negociación. Para identificarlo, se segmentan las cláusulas mayores usando el criterio del verbo. Luego, para clasificar los tipos de tema, se consideran los elementos que preceden al verbo o el mismo verbo si es que no le precediera otra configuración.

Una segunda fuente de datos es el microcorpus. Esta exploración se desarrolla en paralelo a la revisión bibliográfica, con el fin de asegurar el carácter sistémico, funcional y recursivo de la metodología (i.e. del sistema al uso, de la teoría a la descripción). Es esta una especificación que agregamos y que se corresponde con la aproximación cualitativa, basada en el texto (text-based), propuesta y desarrollada por Halliday desde sus primeros trabajos (1989, 1991, 2005a, 2005b, 2005c, 2005d). Se trata de un acercamiento no automatizado al corpus, el que proporciona un sustento empírico a las categorías que emergen de la revisión bibliográfica (Matthiessen 2006), a la vez que una mirada pormenorizada del corpus respecto de la función a describir.

El microcorpus, en este caso, está conformado por una muestra de 10 textos tomados del corpus PUCV-2006 (www.elgrial.cl). Ellos han sido previamente caracterizados, por sus modos de organización discursiva predominante, como argumentativos, descriptivos y narrativos (Parodi, Venegas, Ibáñez y Gutiérrez 2008). Esto importa, pues la variación del perfil probabilístico de las configuraciones lexicogramaticales que realizan la función de tema se asocia a la variación en el modo, constituyente del registro. La Tabla 1, a continuación, presenta la agrupación por modo de organización del discurso y la caracterización cuantitativa del microcorpus.

Tabla 1

Caracterización del corpus

\begin{tabular}{|c|c|}
\hline Modo de organización discursiva & Cantidad de cláusulas \\
\hline Argumentativo & 1.329 \\
\hline Narrativo & 2.073 \\
\hline Descriptivo & 583 \\
\hline
\end{tabular}


Como puede observarse en la Tabla 1, la unidad usada para cuantificar el corpus es la cláusula, esto pues el sistema de tema opera en dicho nivel del rango. Sobre el número de cláusulas, se normalizará para caracterizar, en la siguiente etapa de este trabajo, probabilísticamente las configuraciones lexicogramaticales que realizan tema en español.

\subsubsection{Etapa de caracterización}

En un segundo momento, las configuraciones ya identificadas son caracterizadas, de acuerdo a las dimensiones descriptivas, propuestas por la GSF, para el estrato lexicogramatical, esto es, como se muestra en la Figura 5, más arriba: rango, eje, detalle, continuum de la lexicogramática y probabilidad de ocurrencia. Cada una de estas variables permite, como tuvimos oportunidad de profundizar en el marco teórico, dar cuanta de alguna de las características del estrato lexicogramatical. Se definen como se presenta a continuación.

a) Escala de rango: para caracterizar las opciones léxicas y gramaticales de acuerdo a su comportamiento articulatorio, se utiliza la sistematización propuesta por Halliday (1985) y Halliday y Matthiessen (2004) y especificada por Gutiérrez (2007) para el español. Desde aquí se comprende, tal como lo muestra la Figura 6, que el morfema es la unidad menor y la cláusula la mayor, siendo la palabra y el grupo o frase las unidades de transición.

Figura 6

Escala de rango (adaptada de Halliday 1985)

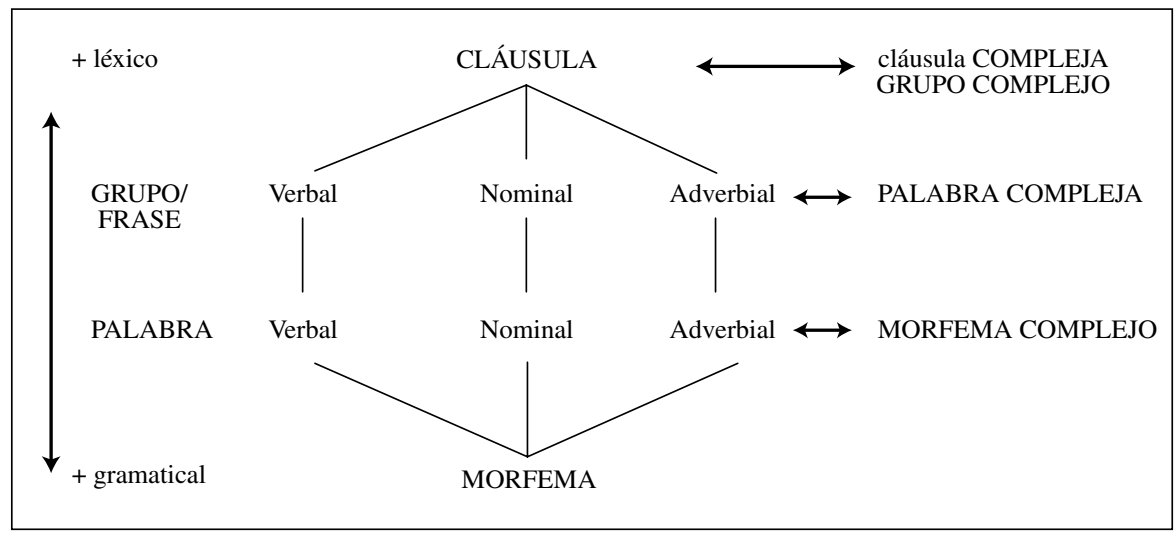

Como notamos en la Figura 6, las unidades pueden caracterizarse como más o menos lexicalizadas, de este modo, el morfema es la unidad más gramaticalizada y la cláusula, la más lexicalizada. El comportamiento del continuum de la lexicogramática es fractal, a consecuencia de lo cual se replica al interior de cada una de las unidades del rango (Huddleston 1965; Hudson 1967; Berry 1977).

b) Continuum de la lexicogramática: el continuum toma la forma de una escala de detalle que posibilita que las unidades, clases y subclases de este estrato 
sean caracterizadas como ítems más léxicos o más gramaticales con una zona intermedia para ítems que comparten características de ambos polos, originando paradigmas léxicos, paradigmas gramaticales y paradigmas intermedios (i.e. semigramaticalizados o semilexicalizados). El paradigma gramatical consta de un conjunto limitado de unidades recurrentes, altamente generales, abstractas, fijas y regulares (Matthiessen 1995; Halliday y Matthiessen 2004) que permiten establecer las regularidades de una lengua. Si bien las configuraciones lexicogramaticales, en este paradigma, son optativas, no son mutuamente excluyentes. Un paradigma léxico, en cambio, se caracteriza por ser un sistema que agrupa ítems con contenido léxico, que si bien no son recurrentes, conforman, por el principio de semejanza de familias (Halliday y Matthiessen 2004), propuesto por Wittgenstein $(1982 ; 2002)$, grupos de configuraciones léxicas que pueden ser elegidas alternativamente de modo excluyente (Matthiessen 1995). Los términos, en este conjunto, son altamente específicos y concretos (Halliday 2002b; Halliday y Matthiessen 2004). En este sentido, el léxico debe ser entendido como un mayor grado de detalle (i.e. delicacy) respecto de la gramática (Hasan, 1984). La Figura 7, que aparece a continuación, sintetiza las diferencias entre ambos paradigmas.

Figura 7

Principales diferencias entre el paradigma léxico y el paradigma gramatical

\begin{tabular}{|l|l|}
\hline Lexical & Gramatical \\
\hline Específico o detallado & General \\
\hline Concreto & Abstracto \\
\hline Opcional & Obligatorio \\
\hline No recurrentes & Recurrentes \\
\hline Abierto & Cerrado \\
\hline
\end{tabular}

c) Caracterización por probabilidades de ocurrencia: las opciones tienen diferentes probabilidades de ocurrencia, dependiendo del contexto de situación en que han sido empleadas. El cálculo de las probabilidades y la consecuente determinación del perfil probabilístico es el que permite dar cuenta del significado como variación, por ello, este dato constituye una condición de la descripción sistémico-funcional. Para realizar el rastreo de la frecuencia -absoluta- y el consecuente cálculo de la probabilidad -frecuencia relativa-, con que cada configuración lexicogramatical es instanciada, constituye un trabajo de corpus basado en el corpus (Caffarell et al. 2004; Halliday 2005a, 2005c, 2005d; Tognini-Bonelli 2001). Sin embargo, en este estudio solo exploramos la probabilidad que las configuraciones tienen de ser instanciadas, utilizando, como se ha dicho, un microcorpus, etiquetado manualmente y diversificado por la variable modo del registro, específicamente, modo de organización discursiva, como se mostró anteriormente en la Tabla 1. Dado que trabajamos sobre la base de una representación sistémica, en la cual el término de un sistema puede ser la condición de entrada a otro sistema menor, el método estadístico que utilizamos es el de probabilidades condicionadas, más 
precisamente, condicionadas a cada término de la red. Para determinar si la variación entre las probabilidades de ocurrencia es estadísticamente significativa se utiliza la comparación de proporciones Z (normal estandarizada), estimándose un error global del $3 \%$.

\section{Resultados}

A continuación se exponen los resultados por cada uno de los objetivos.

\subsection{IDENTIFICACIÓN}

Sobre la base de la revisión bibliográfica de gramáticas del español (RAE 1973; Gili Gaya 1979; Seco 1980; Hernández 1986, 1995; Gómez y Peronard 1988; Bosque 1990; Di Tullio 1997; Porto 1991; Matte 1995a, 1995b; Marcos et al. 1998; Alarcos 1999; Carrasco 1999; Escandell 1999; Garrido 1999; Gómez 1999; Pena 1999; Pérez 1999; Ridruejo 1999; Rojo y Veiga 1999; Serrano-Dolader 1999), de estudios lingüísticos (Palmer 2001; Kerbrat-Orecchioni 1997; Calsamiglia y Tusón 1999; Klinge y Hoeg 2005) y de la exploración de precorpus, hemos identificado cinco tipos de tema en español, tales son, como lo muestra la red sistémica de la Figura 8, tema lógico, tema lógico-topical, tema topical, tema intertopical y tema interpersonal.

\section{Figura 8}

Red sistémica para función de Tema en español

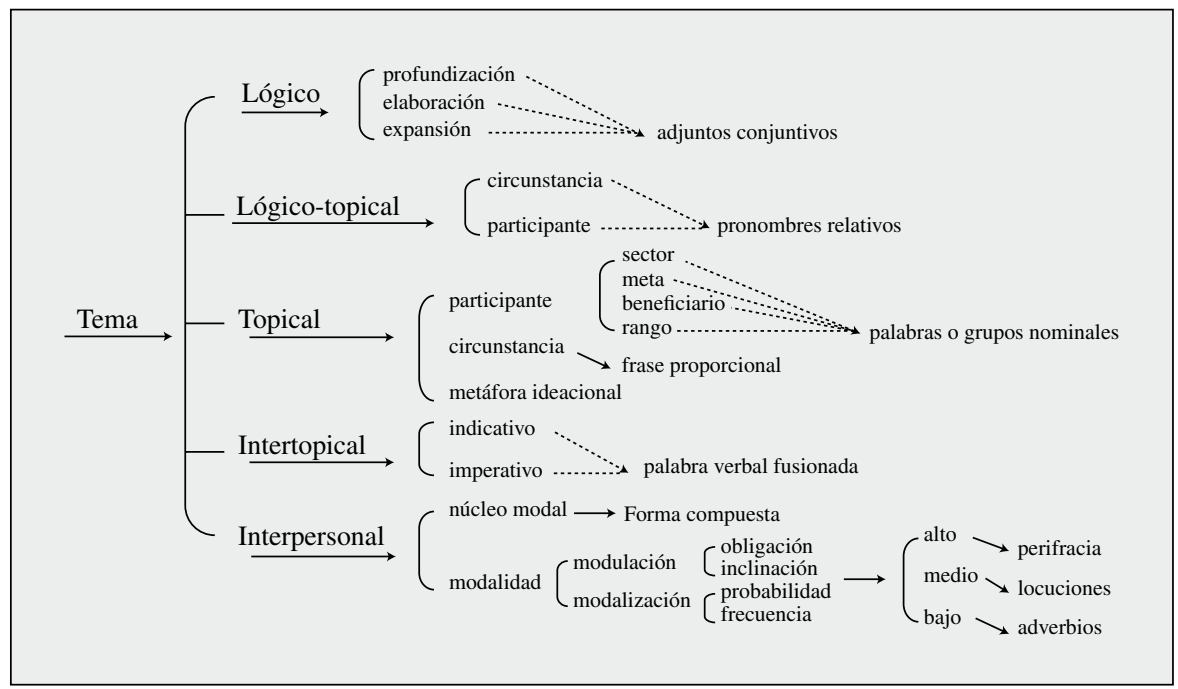

Dado que la representación del mundo es negociada y textualizada para su intercambio, resulta razonable pensar que los elementos de naturaleza topical se mezclen con los de naturaleza textual e interpersonal. De hecho, en el español, muchas 
realizaciones, a nivel de la estructura sintáctica, aglutinan estos diferentes tipos de significado. El aglutinamiento se produce en el marco de las reglas combinatorias que la lengua tiene a nivel de estructura sintáctica y, por lo tanto, el orden, en el cual los significados se presentan, no depende de una elección del hablante. Esta última es la razón por la cual no podemos decir que, por ejemplo, en 'cantaron' se tematiza solo el proceso, pues tanto proceso como negociación (i.e. sujeto y finito temporomodal) aparecen juntos y su ocurrencia es simultánea. La situación recién descrita da origen a dos tipos de temas en los que la representación del mundo se subsume con la negociación y con la textualización, estos son, como se muestra en la red sistémica de la Figura 8, tema lógico topical y tema intertopical.

En la idea de esta red, los diferentes tipos de temas se disponen desde los elementos centrados en el hablante, es decir, en su propósito comunicativo, como lo son los lógicos; hasta los centrados en el oyente, es decir, en la interacción con éste, como lo son los interpersonales.

\subsection{CARACTERIZACIÓN}

\subsubsection{Tema lógico}

Se realiza típicamente por un adjunto conjuntivo, es decir, por un elemento que introduce la vinculación de un párrafo con el resto del texto que le precede. Por ejemplo, 'en pocas palabras' en (a):

(a) ¿Quién fue el hombre de Neandertal? Marcellin Boule, profesor del Museo de Historia Natural de París, no se anda con rodeos: un "aspecto brutal", un "cuerpo vigoroso y pesado", una "cabeza huesuda con mandíbulas fuertes en las que todavía se aprecia el predominio de las funciones puramente vegetativas sobre las funcione cerebrales". $\underline{\text { En }}$ pocas palabras: una bestia bruta.

En general, se trata de elementos muy cercanos al paradigma gramatical de la lengua, pero menos gramaticalizados que los que realizan el tema lógico topical. Compárese 'en pocas palabras' en (a) con 'para', tema lógico ejemplificado en (b). Respecto del perfil probabilístico, los temas lógicos tienen mayor probabilidad de ser instanciados en contextos argumentativos, como se observa en el Gráfico 1.

\section{Gráfico 1}

Probabilidad de instanciación del tema lógico

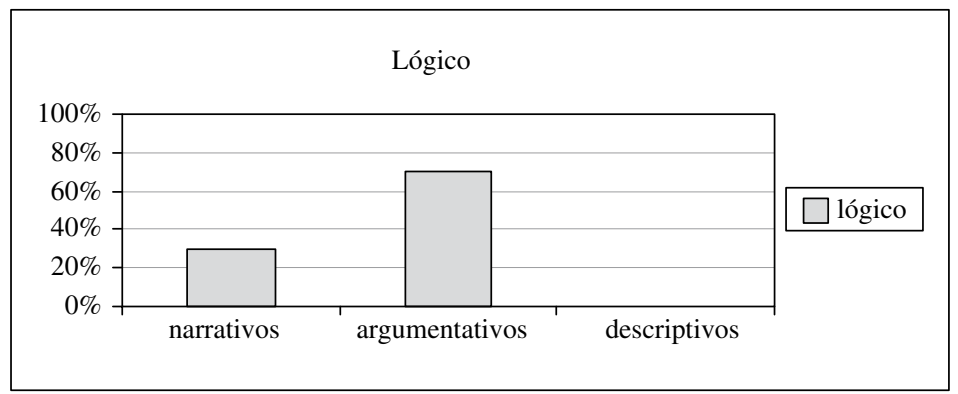


Como se desprende del Gráfico 1, los temas lógicos no se presentan en los contextos descriptivos.

\subsubsection{Tema lógico-topical}

Dado que la metafunción lógica presenta un estatus intermedio entre la metafunción ideacional y la metafunción textual, existe la posibilidad de que aparezca en primera posición un elemento conjuntivo cuya función sea la de evidenciar la relación lógica que existe entre los eventos o procesos en el mundo y no entre los fragmentos en el texto. Esto es lo que llamamos tema lógico topical. El tema lógico-topical se realiza típicamente, a nivel de estructura sintáctica, por una conjunción, como 'para' en el ejemplo (b).

(b) deben seguirse los detalles de dichas plantillas para el envío de originales.

También se considerará tema lógico-topical a los pronombres relativos que, de una parte, remiten a algún participante de la ideacionalidad y de otra, a través de la misma correferencia, operan como un mecanismo de cohesión, por ejemplo 'que' en el ejemplo (c).

(c) Todo académico que ingrese a la Universidad será nombrado en alguna de las jerarquías académicas que establece el artículo 9 de este reglamento.

En concordancia con su naturaleza ideacional, los temas lógico-topicales presentan mayor probabilidad de ocurrencia en los contextos narrativos, tal como lo muestra el Gráfico 2.

Gráfico 2

Probabilidad de instanciación del tema lógico-topical

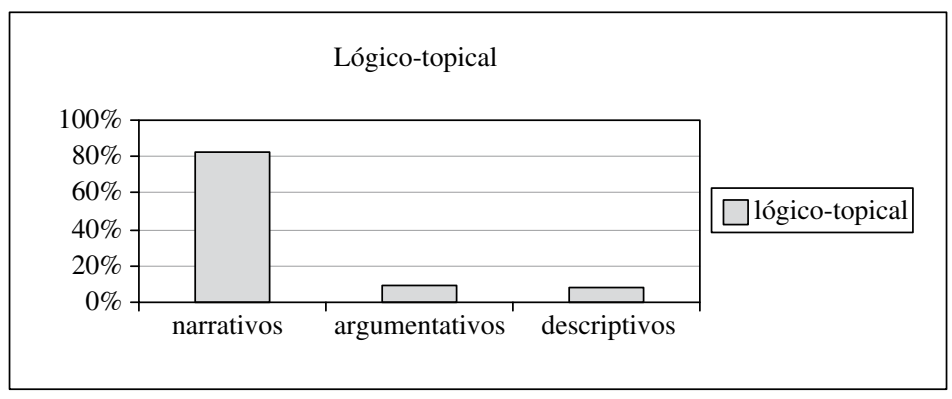

\subsubsection{Tema topical}

Se llama topical al tema si el elemento que ocupa la primera posición en la cláusula corresponde a alguna de las funciones de la ideacionalidad. Si bien en el marco de la metafunción ideacional hay tres tipos de funciones: participantes, proceso y circunstancias, solo dos de ellas pueden ocupar posición temática, como muestra la 
red sistémica de la Figura 8, estas son los participantes y las circunstancias. La función de proceso no ocupa la posición temática pues el comportamiento articulatorio y aglutinante del español hace que los eventos asociados a la representación del mundo siempre aparezcan negociados, como 'reforzarás' en el ejemplo (d).

(d) Reforzarás los contenidos mínimos obligatorios para la PSU.

En efecto, la palabra verbal 'reforzarás' está formada de un morfema lexical que da forma al contenido ideacional del verbo, i.e. 'reforzar' y un morfema gramatical, dando forma a los significados interpersonales, i.e. sujeto gramatical de segunda persona singular, finito temporal de presente y modo indicativo.

3.2.3.1. Participantes: un tema participante a nivel de la estructura funcional puede corresponder al actor (ejemplo (e)), a la meta (ejemplo (f)), al rango (como en el ejemplo (g)) o al beneficiario (ejemplo (h)).

(e) El tequila es un destilado originario de la ciudad del mismo nombre en el estado de Jalisco, México.

(f) El primer envío del original se realizará en formato pdf.

(g) Una hermosa canción fue cantada por John Lennon.

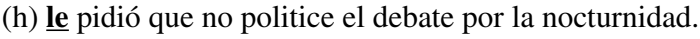

A nivel de la estructura sintáctica, estas funciones pueden realizarse en palabras, grupos o cláusulas de naturaleza nominal, así, por ejemplo, en (f) el participante meta es un grupo nominal y en (h) el beneficiario es una palabra de naturaleza nominal. Los grupos y las cláusulas, siguiendo la escala de rango propuesta por Gutiérrez (2007), son consideradas estructuras más lexicalizadas; el caso del participante realizado por una palabra de naturaleza nominal, sin embargo, puede ser más gramaticalizado si se realiza a través de un pronombre, como, por ejemplo en (h) y más lexicalizado, a través de un nombre, como se presenta en (e) o (f).

Para considerar que el participante actor es tema topical, este debe estar lexicalizado a través de una palabra o grupo de naturaleza nominal, como en el ejemplo (g). Precisamente en (g), el sujeto lógico o actor no coincide con el sujeto gramatical, por lo que es fácil distinguir que se trata de un tema topical. En cambio, si los dos tipos de sujeto coinciden, se utilizará el criterio de la mayor o menor lexicalización para decidir si lo que está tematizado es solo el actor o es el actor y el sujeto gramatical. Así, mientras más lexicalizado más cercano la ideacionalidad y mientras más gramaticalizado, por ejemplo, realizado a través de un pronombre como 'nosotros' en (i), el tema será intertopical o tema que realiza simultáneamente al sujeto lógico y al énfasis del sujeto modal.

(i) Nosotros no vamos a establecer responsabilidades inmediatas.

3.2.3.2. Circunstancias: El tema topical circunstancias se realiza en la estructura sintagmática en una palabra, grupo o cláusula de naturaleza adverbial, como por ejemplo 'con estas herramientas' en ( $\mathrm{j}$ ).

(j) Con estas tres herramientas se puede montar cualquiera de los muebles en kit. 
Tal como se evidencia a través del Gráfico 3, las circunstancias como tema tienen mayor probabilidad de instanciarse en contextos narrativos.

Gráfico 3

Probabilidad de instanciación del tema topical

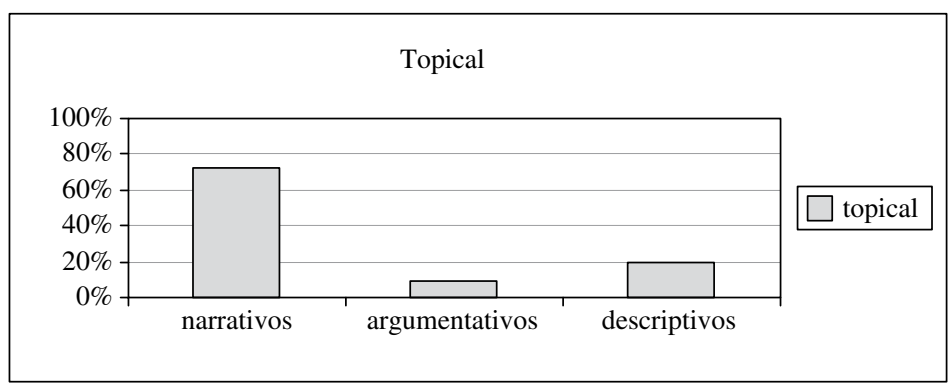

De hecho, la probabilidad disminuye significativamente en los modos argumentativos y descriptivos, tal como ocurría con el tema lógico-topical, según se evidenció a través del Gráfico 2.

\subsubsection{Tema intertopical}

Observemos los siguientes ejemplos:

(k) Conviene no obsesionarse

(1) Nosotros buscamos gente que quiera trabajar

En el primer caso, ejemplo (k), se trata de una palabra en la que converge un morfema lexical que realiza el contenido ideacional de un proceso y morfema, más gramaticalizado, que realiza las subfunciones asociadas al núcleo modal o núcleo de negociabilidad de la cláusula, i.e. sujeto gramatical y finito temporal y modal. En este caso, no podríamos clasificar el tema como ideacional, bajo el criterio de que es el elemento funcional que ocupa la primera posición. Esto, pues tal posición no proviene de una elección del hablante, sino que de una restricción del comportamiento articulatorio del español. En el segundo caso, ejemplo (1), la palabra nominal pronombre realizando al actor o sujeto lógico coincide con el sujeto gramatical, ya realizado por la flexión del verbo y lo enfatiza, por tanto, como se había señalado al tratar el tema topical participante, a este tipo de tema se le considera intertopical.

A nivel de la estructura sintáctica, el tema intertopical tiene, entonces, dos posibles realizaciones: una palabra de naturaleza verbal o una palabra de naturaleza nominal. Esta última corresponde a una realización más cercana al paradigma gramatical de la lengua; la otra, la palabra de naturaleza verbal, mantiene una posición intermedia, por cuanto, como se explicitó, está compuesta de una unidad lexicalizada y una gramaticalizada. 
Este tipo de tema presentó una mayor probabilidad de ocurrencia en los contextos descriptivos, tal como se grafica en 4.

Gráfico 4

Probabilidad de instanciación del tema intertopical

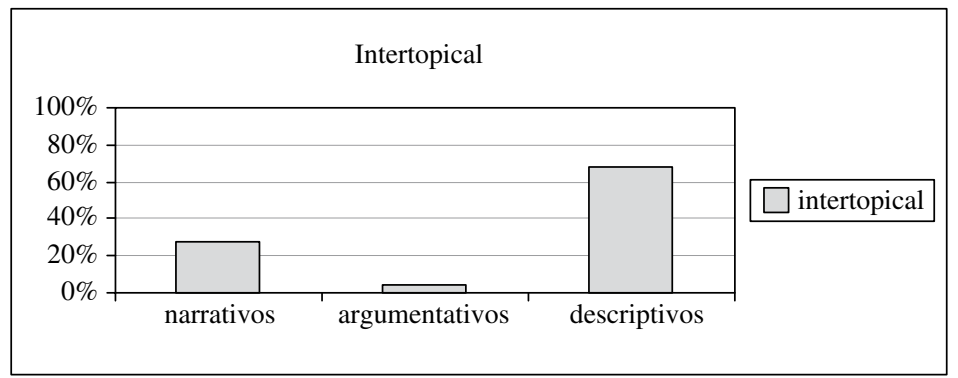

\subsubsection{Tema interpersonal}

Este tipo de tema presenta dos opciones de realización a nivel de la estructura funcional: en el núcleo modal y en el adjunto de modalidad. Si se trata del núcleo modal, este se realiza en el marco de un grupo verbal, como por ejemplo en (m), y si se trata de un adjunto de modalidad, la realización prototípica a nivel sintáctico es en una palabra o grupo de naturaleza adverbial y, dentro de ellos, en los que significan modo, ejemplo (n).

(m) Tenía que pasar tarde o temprano

(n) Probablemente desapareció para siempre

Como puede notarse en los ejemplos, la realización a través de adverbio de modo es más cercana al paradigma léxico que la realización a través del verbo auxiliar en un grupo verbal, tal como lo muestra la Figura 9.

Figura 9

Red del tema interpersonal

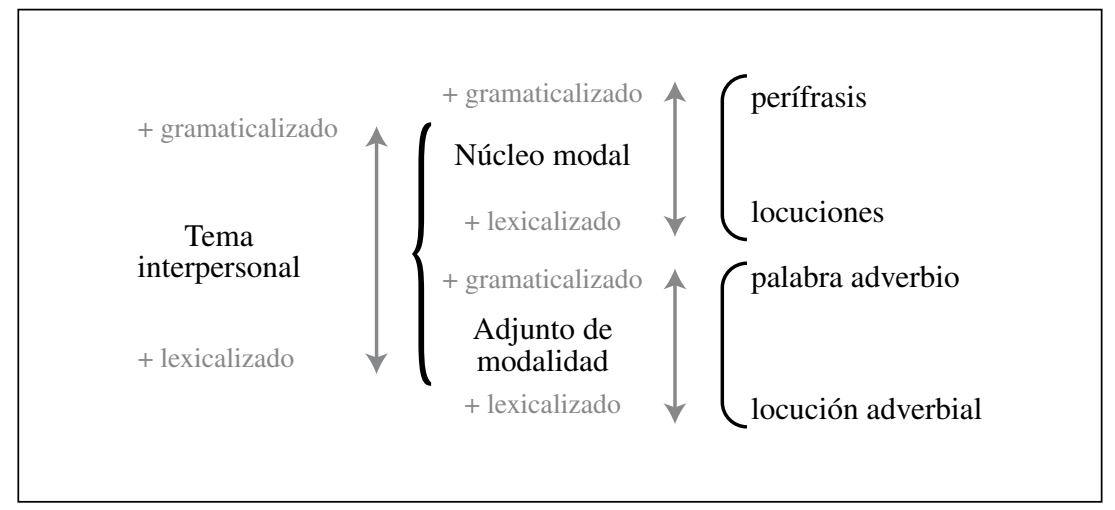


Sin embargo, se debe recordar que hay un mayor nivel de detalle, pues de una parte, desde el rango para el español (Gutiérrez, 2007), los grupos verbales se gradúan de acuerdo al continuum de la lexicogramática, desde los más gramaticalizados, que serían las perífrasis verbales hasta los más lexicalizados: las locuciones verbales. De otra, los adverbios de modo que realizan tema interpersonal corresponden, en general, a adverbios terminados en '-mente', morfema de modo altamente gramaticalizado que hace que los grupos adverbiales adquieran el rasgo de mayor lexicalización.

Los temas interpersonales presentan, según el contexto de observación usado en este estudio, la variación que se exhibe en el Gráfico 5.

Gráfico 5

Probabilidad de instanciación del tema interpersonal

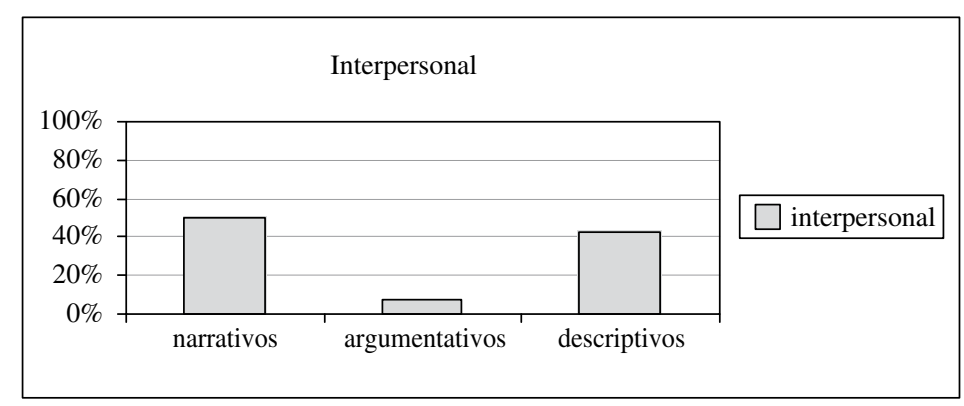

Tal como es posible apreciar en el Gráfico 5, el tema interpersonal presenta una probabilidad de ocurrencia semejante en contextos narrativos y en contextos descriptivos, con una diferencia que no es estadísticamente significativa. Es interesante resaltar que este es el único tipo de tema que presenta este comportamiento probabilístico, pues los otros cuatro muestran una clara preferencia por alguno de los modos discursivos usados acá como contexto de observación.

\section{CONCLUSIONES}

En español la realización de la función de tema presenta las siguientes características:

Respecto del rango, se trata de una función cuya realización siempre se ubica en un nivel superior al morfema. Esto, probablemente se deba a que la articulación en español impone una ubicación rígida para la integración de los morfemas en palabras y, en consecuencia, un morfema en primera posición no es una elección del hablante para especificar sus propósitos comunicativos.

La elección de primera posición fue abordada en este trabajo a nivel de cláusula y, por tanto, una interesante proyección es estudiar el comportamiento temático en los complejos clausulares o, aún más ambiciosamente, a nivel de los move retóricos que caracterizan a los tipos textuales. Esta última proyección implicaría diversificar el corpus de acuerdo a otra de las opciones de la variable de modo del registro: el tipo de texto. Con ello, se avanzaría hacia la descripción de una gramática del texto, 
más allá del estudio de los mecanismos de cohesión y coherencia, que, se ocupara de describir las siluetas textuales o formas prototípicas de textualizar un representación del mundo.

Respecto del continuum de la lexicogramática fue posible constatar que, excepto para el tema lógico, cada tipo de tema presenta una opción de realización más gramaticalizada y una más lexicalizada. A su vez, en términos generales, entre ellas, es posible reconocer que los temas lógico y lógico-topical tienen una realización más gramaticalizada que los temas intertopical y topical, en tanto que el tema interpersonal correspondería a una categoría intermedia del continuum.

Respecto de la variación por contexto, el que acá fue explorado desde la variable de modo fue el modo de organización discursiva. Según este, los tipos de tema variaron su perfil probabilístico, de modo que cada tema resultó por asociarse a solo uno de los modos de organización discursiva. Así el tema lógico a la argumentación; el tema lógico-topical, el topical y el interpersonal a la narración; el intertopical y el interpersonal a la descripción. Como notamos, el tema interpersonal domina en dos contextos: narración y descripción. Tres proyecciones se desprenden de este conclusión. En primer lugar y como ya se señaló, se podría diversificar el corpus según otra de las subvariables del modo. En segundo lugar, se podría ampliar el corpus y automatizar su análisis. Asimismo, el corpus podría ser depurado al tomar solo las secuencias narrativas, descriptivas o argumentativas y no los textos completos en los que, si bien una domina, suelen instanciarse todas. En otra línea, podrían utilizar estos resultados como rasgos para caracterizar la argumentación, la narración y la descripción desde los rasgos lingüísticos.

\section{OBRAS CITADAS}

Alarcos, Emilio. 1999. Gramática de la lengua española. Madrid: Espasa Calpe.

Arus, Jesús. 2006. Perspectiva sistémico-funcional de los usos de 'se' en español. Revista signos, 39 (61), 131-159.

Berry, Margaret. 1977. An introduction to systemic linguistics 1: Structures and systems. Sydney: B.T. Batsford.

Biber, Douglas, Conrad, S. y Reppen, R. 1998. Corpus linguistics. Investigating language structure and use. Cambridge: Cambridge University Press.

Bosque, Ignacio. 1990. Tiempo y aspecto en español. Madrid: Cátedra.

Bosque, Ignacio y Demonte, V. 1999. Gramática descriptiva de la lengua española. Madrid: Espasa Calpe.

Caffarell, Alice. 1995. Approaching the french clause as a move in dialogue: Interpersonal Organization. En R. Hasan y P. Fries (Eds.), On subject and Theme: A discourse functional perspective (pp. 1-49). Amsterdam: John Benjamins.

Caffarell, Alice. 2004. Metafunctional profiles of the grammar of French. En A. Caffarell, J. Martin y C. Matthiessen (Eds.), Language typology. A functional perspective (pp. 77138). Amsterdam: John Benjamins.

Caffarell, Alice, Martin, Jim y Matthiessen, Christian. 2004. Introduction: Systemic functional typology. En A. Caffarell, J. Martin y C. Matthiessen (Eds.), Language typology. A functional perspective (pp. 1-76). Amsterdam: John Benjamins.

Calsamiglia, Helena y Tusón, Amparo. 1999. Las cosas del decir. Manual de análisis del discurso. Barcelona: Ariel. 
Carrasco, A. 1999. El tiempo verbal y la sintaxis oracional. La consecutio temporum. En I. Bosque, Ignacio y Demonte, Violeta (Dirs.), Gramática descriptiva de la lengua española (pp. 3061-3130). Vol. 2. Madrid: Espasa Calpe.

De Vega, Manuel. 2005. Lenguaje, corporeidad y cerebro: Una revisión crítica. Revista signos, 38 (58), 157-176.

Di Tullio, Ángela. 1997. Manual de gramática del español. Desarrollos teóricos. Ejercicios, soluciones. Buenos Aires: Edicial.

Duc, M. 2004. Metafunctional profile of the grammar of Vietnamese. En A. Caffarell, J. Martin y C. Matthiessen (Eds.), Language typology. A functional perspective (pp. 397-428). Ámsterdam: John Benjamins.

Escandell, $M^{a}$ Victoria. 1999. Los enunciados interrogativos. Aspectos semánticos y pragmáticos. En I. Bosque y V. Demonte (Dirs.), Gramática descriptiva de la lengua española (pp. 3929-3991). Vol. 3. Madrid: Espasa Calpe.

Fairclough, Norman. 1995. Critical discourse analysis: The critical study of language. Harlow: Pearson Education.

Firth, J.R. 1968a. Linguistic analysis and translation. En F. Palmer (Ed.), Selected papers of J.R. Firth (pp. 74-83). Londres: Indiana University.

—. 1968b. Linguistic and translation. En F. Palmer (Ed.), Selected papers of J.R. Firth (pp. 84-95). Londres: Indiana University.

- 1968c. Ethnografic analysis and language with reference to Malinowski's View. En Palmer, F. (Ed.) Selected papers of J.R. Firth $1952-59$ (pp. 168-205). London: Indiana University Press.

Garrido, J. 1999. Los actos de habla. Las oraciones imperativas. En I. Bosque y V. Demonte (Dirs.), Gramática descriptiva de la lengua española (pp. 3879-3928). Vol. 3. Madrid: Espasa Calpe.

Gili Gaya, Samuel. 1979. Curso superior de sintaxis española. Barcelona: Biblograf.

Gómez, Luis y Peronard, Marianne. 1988. El lenguaje Humano. Léxico fundamental para la iniciación lingüística. Valparaíso: Universidad Católica de Valparaíso. Gómez, L. (1999). Los verbos auxiliares. Las perífrasis verbales de infinitivo. En I. Bosque y V. Demonte.

Gutiérrez, R.M. 2007. Realización lexicogramatical del sistema semántico de la modulación: Una aproximación a la descripción sistémico-funcional del español. Tesis doctoral. Pontificia Universidad Católica de Valparaíso, Valparaíso, Chile.

- 2008. El género manual en las disciplinas académicas: Una caracterización desde el sistema de la obligación. Rev. Signos, Valparaíso, v. 41, n. 67.

—. 2010. Oralidad, escritura y especialización: una caracterización desde el sistema de la obligación, Revista de Lingüística Aplicada, 48(1), pp. 105-132.

Demonte, V. (Dir.). Gramática descriptiva de la lengua española (pp. 3253-3322). Vol. 2. Madrid: Espasa Calpe.

Halliday, M.A.K. 1982. El Lenguaje como Semiótica Social. La Interpretación Social del Lenguaje y del Significado. México: Fondo de Cultura Económica.

- 1985. An Introduction to Functional Grammar. London: Arnold.

—. 1989. Spoken and Written Language. Hong Kong: Oxford University Press.

1991. Corpus studies and probabilistic grammars. En K. Aijmer y B. Altenberg (Eds.), English corpus linguistics. Studies in hounor of Jan Svartvik (pp. 31-43). London: Longman.

1992. Language as a system and language as a instance: the corpus as a theoretical construct. En J. Svartvik (Ed.), Directions in corpus linguistics (pp. 61-77). New York: Mouton de Gruyter.

2002a. On Grammar and Grammatics (1996). En J. Webster (Ed.), On grammar (pp. 384417). London: Continuum. 
- 2002b. Categories of the Theory of Grammar (1961). En J. Webster (Ed.), On grammar (pp. 37-94). London: Continuum.

. 2002c. Class in Relation to the Axes of Chain and Choice in Language (1963). En J. Webster (Ed.), On grammar (pp. 95-105). London: Continuum.

2002d. How Do You Mean? (1992). En J. Webster (Ed.), On grammar (pp. 352- 368). London: Continuum.

2003a. On the "Architecture" of Human Language. En J. Webster (Ed.), On language and linguistics (pp. 1-29). London: Continuum.

2003b. A brief sketch of systemic grammar (1969). En J. Webster (Ed.), On language and linguistics (pp. 180-184). London: Continuum.

2003c. Ideas about language (1977). En J. Webster (Ed.), On

2003d. A brief sketch of systemic grammar (1969). En J. Webster (Ed.), On language and linguistics (pp. 180-184). London: Continuum. Language and linguistics (pp. 92-115). London: Continuum

- 2004. The Language of Science. London: Continuum.

2005a. Linguistics and machine translation (1962). En J. Webster (Ed.), Computational and quantitative studies (pp. 20-35). London: Continuum.

- 2005b. Corpus studies and probabilistic grammar (1991). En J. Webster (Ed.), Computational and quantitative studies (pp. 63-75). London: Continuum.

- 2005c. Towards probabilistic interpretation (1991). En J. Webster (Ed.), Computational and quantitative studies (pp. 42-62). London: Continuum.

—. 2005d. Quantitative studies and probabilities in grammar (1993). En J. Webster (Ed.), Computational and quantitative studies (pp. 130-156). London: Continuum.

Halliday, M.A.K. y Matthiessen, Christian. 1999. Construing experience through meaning: a language based approach to cognition. London: Cassell.

- 2004. An Introduction to Functional Grammar. London: Arnold.

Halliday, M.A.K. y McDonald, E. 2004. Metafunctional profile of the grammar of the Chinese. En A. Caffarell, J. Martin y C. Matthiessen (Eds.), Language typology. A functional perspective (pp. 305-396 ). Amsterdam: John Benjamins.

Hernández, César. 1986. Gramática funcional del español. Madrid: Gredos.

—. 1995. Nueva sintaxis de la lengua española. Salamanca: Ediciones Colegio de España.

Hjelmslev, Louis. 1974. Prolegómenos a una teoría del lenguaje. Madrid: Gredos.

Hood, Susan. y Martin, Jim. 2005. Invocación de actitudes: El juego de la gradación de la valoración en el discurso. Revista Signos, 38 (58), 195-220.

Huddleston, R. 1965. Rank and depth. Language, 41, 574-586.

Kerbrat-Orecchioni, Catherine. 1997. La enunciación. De la subjetividad en el lenguaje. Buenos Aires: Edicial.

Klinge, A. y Hoeg, H. 2005. Modality. Studies in form and function. London: Equinox.

Marcos, F., Satorre, F. y Viejo, M. 1998. Gramática española. Madrid: Síntesis.

Martin, Jim. 1992. English Text. System and Structure. Philadelphia: John Benjamins.

Martínez, E. 1999. Gramática del discurso: Foco y énfasis en inglés y en español. Barcelona: PPU.

Matte, F. 1995a. Gramática comunicativa del Español. De la idea a la lengua. Vol. 2. España: Edelsa.

. 1995b. Gramática comunicativa del Español. De la lengua a la idea. Vol. 1. España: Edelsa.

Matthiessen, Christian. 1993. Register in the round diversity in a unified theory of register analysis. En M. Ghadessy (Ed.), Register analysis. Theory and practice (pp. 221-292). London: Painter. 
Matthiessen, C. 1995. Lexicogrammatical Cartography: English System. Tokyo: Meadea. Matthiessen, C. 2008. "The architecture of grammar" according to the systemic functional theory of language.

2006. Frequency profiles of some basic grammatical systems: an interim report. En G. Thompson y S. Hunston (Eds.), System and Corpus. Exploring Connections (pp. 103142) London: Equinox.

Moss, Gillian y Chamorro, Daniel. 2008. La enseñanza de la ciencia sin asidero en el tiempo ni en el espacio: análisis del discurso de dos textos escolares, Lenguaje, 36 (1), pp. 87-115.

Moss, Gillian, Natale, Lucía y Oteiza, Teresa. 2009. El lenguaje de los textos escolares: el aprendizaje, la ideología y la formación ciudadana, Revista Documentação De Estudos Em Lingüística Teórica E Aplicada (DELTA), 25, pp. 641-677.

Moss, Gillian. 2010. Textbook language, ideology and citizenship. The case of a history textbook in Colombia, Functions of Language, 17 (1), pp. 71-93.

Moyano, Estela. 2007. Enseñanza de habilidades discursivas en español en contexto preuniversitario: Una aproximación desde la LSF. Revista Signos, 40 (65), pp. 573-608.

Porto Dapena, J.A 1991. Del indicativo al subjuntivo. Madrid: Arco/Libros.

Palmer, F. 2001. Mood and modality. Cambridge: Cambridge University Press.

Pena, J. 1999. Partes de la morfología. Las unidades del análisis morfológico. En I. Bosque y V. Demonte (Dir.), Gramática descriptiva de la lengua española (pp. 4305-4366). Vol. 3. Madrid: Espasa Calpe.

Pérez, M. 1999. El modo en las subordinadas relativas y adverbiales. En I. Bosque y V. Demonte (Dir.), Gramática descriptiva de la lengua española (pp. 3253-3322). Vol. 2. Madrid: Espasa Calpe.

RAE. 1973. Esbozo de una nueva gramática de la lengua española. Madrid: Espasa Calpe.

Ridruejo, E. 1999. Modo y modalidad. El modo en las subordinadas sustantivas. En I. Bosque y V. Demonte (Dir.), Gramática descriptiva de la lengua española (pp. 3209-3251). Vol. 2. Madrid: Espasa Calpe.

Rojo, G. y Veiga, A. 1999. El tiempo verbal. Los tiempos simples. En I. Bosque y V. Demonte (Dir.), Gramática descriptiva de la lengua española (pp. 2867-2934). Vol. 2. Madrid: Espasa Calpe.

Seco, R. 1980. Manual de gramática española. Madrid: Aguilar.

Serrano-Dolader, D. 1999. La derivación verbal y la parasíntesis. En I. Bosque y V. Demonte (Dir.), Gramática descriptiva de la lengua española (pp. 4683-4755). Vol. 3. Madrid: Espasa Calpe.

Steiner, E. y Teich, E. 2004. Metafunctional profile of the grammar of German. En A. Caffarell, J. Martin y C. Matthiessen (Eds.), Language typology. A functional perspective (pp. 139183). Amsterdam: John Benjamins.

Teruya, K. 2004. Metafunctional profile of the grammar of Japanese. En A. Caffarell, J. Martin y C. Matthiessen (Eds.), Language typology. A functional perspective (pp. 185-251). Amsterdam: John Benjamins.

Tognini-Bonelli, Elena. 2001. Corpus linguistics at work. Amsterdam: John Benjamins.

Wittgenstein, Ludwig. 1982. Últimos escritos sobre filosofía de la psicología. Estudios preliminares para la parte II de investigaciones filosóficas. Madrid: Tecnos.

—. 2002. Investigaciones filosóficas. Barcelona: Crítica. 